\title{
Chapter 11 \\ Estimating Currents and Electric Fields at Low Latitudes from Satellite Magnetic Measurements
}

\author{
Patrick Alken
}

\begin{abstract}
Low-latitude ionospheric electric currents produce prominent signatures in the magnetic field measurements made by low Earth-orbiting satellites. Analyzing these magnetic signatures not only provides insight into the currents themselves, but also many other important and interesting phenomena in the low-latitude ionosphere and thermosphere. The low-latitude currents are modulated by thermospheric winds, so attaining a global knowledge of the spatial structure of the currents can give insight into the neutral tidal harmonics present at ionospheric altitudes. Furthermore, the equatorial electrojet (EEJ) current is driven by an equatorial electric field which in turn is generated by a dynamo process. This electric field is additionally responsible for the vertical plasma fountain and equatorial ionization anomaly at low-latitudes. Magnetic measurements of the EEJ, therefore, allows the study of low-latitude plasma motion in the $\mathrm{E}$ and $\mathrm{F}$ regions of the ionosphere. This chapter will present techniques developed for processing magnetic measurements of the EEJ to extract information about the low-latitude currents and their driving electric fields. This chapter will present a line current approach to recover the EEJ current strengths, with an emphasis on cleaning the satellite data and minimizing magnetic fields from other internal and external sources. The electric fields will be determined using a combination of physical modeling and fitting the EEJ current strengths from the satellite measurements.
\end{abstract}

\subsection{Introduction}

This chapter will be concerned with the calculation of ionospheric current flow and electric fields at low-latitudes, using magnetic field measurements from low Earth orbiting (LEO) satellite missions, such as Swarm (Friis-Christensen et al. 2006). In the ionosphere, neutral particles are ionized by solar extreme ultraviolet (EUV) radiation. The resulting charged plasma then interacts with the Earth's electromagnetic

P. Alken $(\varangle)$

University of Colorado at Boulder, Boulder, CO, USA

e-mail: alken@colorado.edu 
field, neutral wind field, gravitational forces, and pressure-gradient forces. Each of these forces drives electric current flow, which has complex spatial and temporal structure. In the ionospheric E-region, which extends from about 90 to $120 \mathrm{~km}$ altitude, ion-neutral collisions are significant, while the electrons are mostly frozen to magnetic field lines. The frictional forces between the ions and the neutral wind field drive current which in general is not divergence free. Therefore, polarization electric fields build up globally to ensure divergence-free current flow. At low and mid-latitudes, the large-scale current system resulting from this ion-neutral coupling is called Solar-quiet (Sq). "Solar" refers to the current system's dependence on solar local time, since ionospheric conductivity at mid-latitudes peaks during daytime hours and diminishes during the night. The term "quiet" indicates geomagnetically quiet conditions, since during strong geomagnetic storms, the Sq system can experience large perturbations. At the magnetic equator, the horizontal geometry of the field lines leads to an enhanced zonal current called the equatorial electrojet (EEJ). An eastward component of the electric field at the equator, when coupled with the northward geomagnetic field, will drive vertical drift of electrons. Above about $120 \mathrm{~km}$ altitude, the Hall conductivity decreases substantially, since the reduced density of neutral particles result in less ion-neutral collisions, and the ions are essentially free to move with the electrons. This effect causes a nonconducting layer at the top of the E-region, and so the charge will accumulate at about $120 \mathrm{~km}$ altitude near the magnetic equator. This charge accumulation will cause a strong vertical polarization electric field which will drive zonal electron drift. Because the vertical polarization electric field is typically about 10 times stronger than the eastward component, the zonal $\mathbf{E} \times \mathbf{B}$ drift results in a strong current system, the EEJ. The EEJ has prominent magnetic signatures in both LEO satellite and ground observatory data, offering a convenient means of studying equatorial E-region dynamics. Since the EEJ is driven by the eastward component of the equatorial electric field (EEF), and is modulated by the neutral wind field, studying its magnetic signature reveals a lot of important information about the equatorial electrodynamics and also tidal features in the neutral winds.

This chapter will discuss recent methods of fitting an equivalent current model, based on line current geometry, to Swarm satellite magnetic measurements in order to recover the EEJ current strength flowing in the E-region. Line current methods have long been applied to studies of both the equatorial electrojet (Lühr et al. 2004; Alken et al. 2013a, 2014) and polar electrojets (Olsen 1996; Ritter et al. 2004; Vennerstrom and Moretto 2013; Aakjær et al. 2016). Since the electrojets typically follow lines of constant magnetic latitude, they have relatively simple spatial flow patterns, and line current models are particularly suitable for their study. For current systems which have more spatial complexity, such as Sq, pressure-gradient currents, interhemispheric field-aligned currents (IHFAC), and high-latitude field-aligned currents (FAC), it is necessary to utilize other methods to determine equivalent current flow, such as spectral methods (Fiori and Boteler 2018) or spherical elementary current systems (SECS) (Vanhamäki et al. 2018). This chapter will follow the work of Alken et al. $(2013 \mathrm{a}, 2014)$ in order to define line currents which follow lines of constant quasi-dipole (QD) latitude in the equatorial region to estimate realistic EEJ equivalent 
current flow. These current estimates will then be inverted using a physics-based modeling procedure in order to recover the eastward component of the equatorial electric field.

\subsection{Satellite Data Preprocessing}

Any scientific data collection system, such as LEO satellite magnetometers, can contain erroneous measurements not related to the physical system under study. Therefore in order to recover accurate low-latitude currents and electric fields from satellite measurements, the magnetic data must be carefully preprocessed to isolate the signal of interest and remove other contamination as much as possible. Satellite magnetic data can be preprocessed in many different ways. The approach used in this chapter closely follows previous work in studying magnetic perturbations from lowlatitude ionospheric current systems (Alken and Maus 2010a, b; Alken et al. 2013b, 2015). We will focus only on the scalar field measurements in this chapter, since they contain enough information at low-latitudes to determine equivalent current flow in the E-region. First, the satellite data are separated into half-orbital tracks, extending from the south to north pole or vice versa. This is convenient since the lowlatitude currents are analyzed on an orbit-by-orbit basis. In order to identify potential problems with the measurements, a convenient quantity to examine is the along-track root-mean-square (rms) difference between the scalar magnetic field measurements and a recent main field model, typically from $60^{\circ} \mathrm{S}-60^{\circ} \mathrm{N}$ QD latitude. The alongtrack rms is computed only at mid and low latitudes, since high-latitude scalar field data are influenced heavily by polar electrojets which are difficult to model and would add large contributions to the rms differences. Tracks with a large rms difference in the above latitude range are discarded from analysis. A typical threshold for rms scalar differences is $150 \mathrm{nT}$. This threshold is large enough to keep good geophysical data that may be perturbed significantly during geomagnetic storms, but small enough to discard completely erroneous measurements that may occur due to instrument problems, satellite maneuvers, etc. An example of such events is shown in Fig. 11.1. Panel (a) shows scalar field residuals from Swarm A on a single day, 30 January 2014, when orbit maneuvers perturbed the absolute scalar magnetometer (ASM) measurements. Some tracks exhibit up to $1000 \mathrm{nT}$ difference with the main field model at mid-latitudes, which is highly unlikely to be due to real geophysical signal. These data are not flagged in any way in the Level-1b Swarm dataset, and so this type of analysis is required in order to detect such events. Panel (b) shows the remaining scalar field residuals after discarding all data with an along-track rms greater than $150 \mathrm{nT}$. Note the reduced scale on the vertical axis. The remaining tracks differ with the main field model only up to about $20 \mathrm{nT}$ at low and mid-latitudes, which is normal for ionospheric signals. Panel (c) shows a time series of Swarm A scalar residuals from November 2013 until December 2014, plotting residuals below 60 QD latitude. There are a small number of localized perturbations in the residuals, including the event on 30 January 2014. This shows that these events are uncommon, but must 
(a)

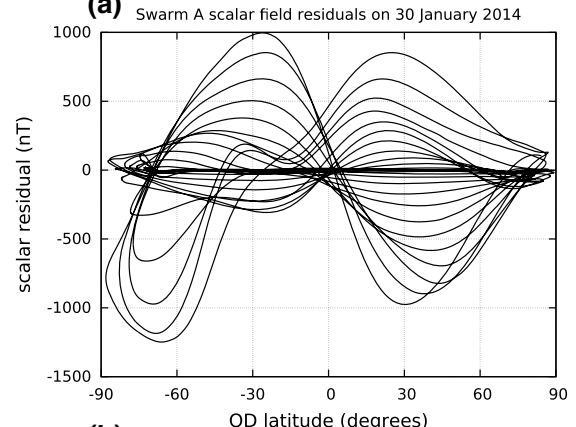

(b)

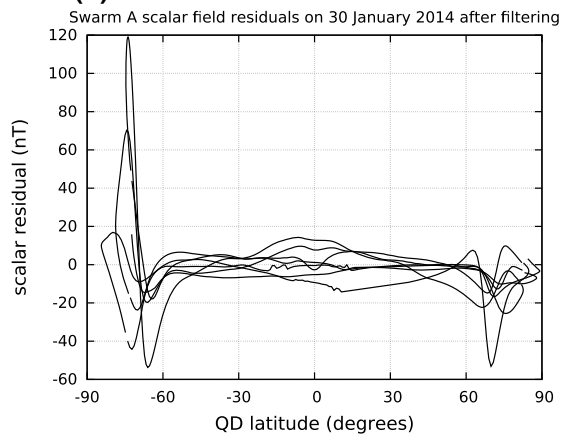

(c)

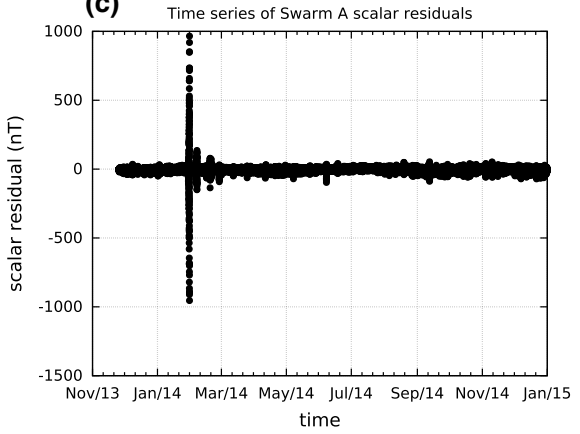

(d)

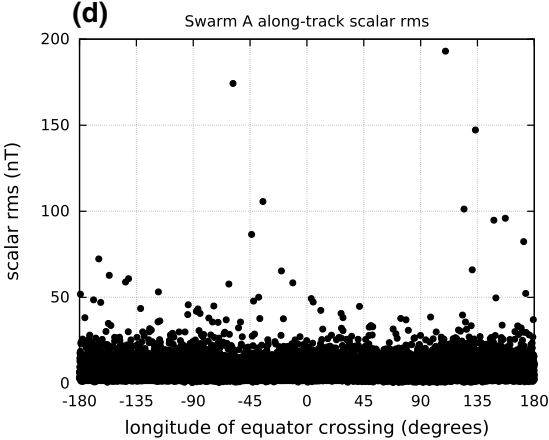

Fig. 11.1 a Scalar residuals for all tracks for Swarm A on 30 January 2014. b Swarm A scalar field residuals after removing tracks with large along-track rms differences with main field model. c Time series of Swarm A scalar residuals from the beginning of the mission to the end of 2014. d Along-track scalar rms values plotted versus the longitude of the geographic equator crossing for same time period as (c)

nevertheless be detected and removed from the data. A useful way to determine an appropriate rms threshold is shown in panel (d), where the along-track rms value is plotted versus the longitude of the satellite's geographic equator crossing, for the same time period as panel (c). Most of the along-track scalar rms values are well below $50 \mathrm{nT}$, and so outliers are fairly straightforward to identify in such a plot. Experience has shown that the along-track rms can easily exceed $100 \mathrm{nT}$ during strong geomagnetic storms, which leads to the choice of $150 \mathrm{nT}$ as a threshold value.

After discarding erroneous measurements as discussed above, the next step is to isolate the ionospheric field signal from other sources in the data. The primary non-ionospheric sources at satellite altitudes are the main field, lithospheric field, and magnetospheric field with its induced counterpart. The main field and its secular variation can be removed using high-quality models such as CHAOS (Finlay et al. 2016) or POMME (Maus et al. 2010). The lithospheric field can similarly be removed using a high-resolution model such as MF7 (Maus et al. 2008). The magnetospheric field can be removed using models of the ring current and tail currents, based on the techniques of Maus and Lühr (2005), Lühr and Maus (2010). Denoting 
the main, crustal, and magnetospheric models as $\mathbf{B}_{\text {core }}, \mathbf{B}_{\text {crust }}$, and $\mathbf{B}_{\text {ext }}$ respectively, an appropriate model of the non-ionospheric sources is given by

$$
\mathbf{B}_{\text {model }}=\mathbf{B}_{\text {core }}+\mathbf{B}_{\text {crust }}+\mathbf{B}_{\text {ext }}
$$

with the scalar residual given by

$$
F^{(1)}=F_{\text {sat }}-\left|\mathbf{B}_{\text {model }}\right|
$$

Here, $F_{\text {sat }}$ is the scalar field measurement made by a LEO satellite. The core and crustal field sources at satellite altitude are generally well represented by current field models, but the magnetospheric sources can vary with complex temporal behavior which is still not completely understood. Therefore while $\mathbf{B}_{\text {ext }}$ will remove most of the external magnetospheric field, some residual could remain. This can be mitigated to some extent by fitting low-degree spherical harmonic models in solar magnetic (SM) and geocentric solar magnetospheric (GSM) coordinates on a track-by-track basis to attempt to further remove any remaining ring and tail current fields (see recent review by Lühr et al. 2016). After the $F^{(1)}$ residual is computed, it will primarily represent the ionospheric field plus its induced counterpart. This is then the starting point to isolate the EEJ contribution to the ionospheric field, so that it can be modeled with line currents, and then used to extract information about the low-latitude electric field.

\subsection{Removing the Sq Field}

The $F^{(1)}$ residual defined in Sect. 11.2 will contain ionospheric signals from Sq, EEJ, inter-hemispheric field-aligned current (IHFAC), polar electrojets (PEJ), highlatitude field-aligned currents (FAC), induced fields originating inside the solid Earth, as well as unmodeled field contributions from the magnetosphere. Since our goal is to recover the EEJ current at low-latitudes, the main concern is removing the $\mathrm{Sq}$ signal from the data, as well as unmodeled magnetospheric ring current fields. To do this, assume that the Sq field and its induced counterpart can be well represented by a magnetic scalar potential for sources internal to the satellite orbit, defined in a spherical coordinate system $(r, \theta, \phi)$ as

$$
V_{i n t}\left(r, \theta, \phi ; g_{n}^{m}\right)=a \sum_{n=1}^{N_{I}} \sum_{m=-1}^{1} g_{n}^{m}\left(\frac{a}{r}\right)^{n+1} Y_{n}^{m}(\theta, \phi)
$$

where $a$ is taken to be an Earth radius of $6371.2 \mathrm{~km}, g_{n}^{m}$ are model coefficients to be determined, $N_{I}$ is the maximum spherical harmonic degree needed to model the $\mathrm{Sq}$ field, and $Y_{n}^{m}(\theta, \phi)$ are defined as 


$$
Y_{n}^{m}(\theta, \phi)=\left\{\begin{array}{cc}
S_{n}^{m}(\cos \theta) \cos (m \phi) & m \geq 0 \\
S_{n}^{|m|}(\cos \theta) \sin (|m| \phi) & m<0
\end{array}\right.
$$

where $S_{n}^{m}(\cos \theta)$ are the Schmidt-normalized associated Legendre functions. The Sq field is normally large-scale without sharp localized features, and so $N_{I}=12$ is a typical choice for the cutoff. Since $V_{\text {int }}$ is fitted to a single satellite orbit, it can be expanded only to spherical harmonic order 1 , since a polar orbiting satellite does not provide enough longitudinal coverage to use higher orders. In order to account for unmodeled magnetospheric fields, we use the external scalar potential

$$
V_{\text {ext }}\left(r, \theta, \phi ; q_{n}^{m}\right)=a \sum_{n=1}^{N_{E}} \sum_{m=-1}^{1} q_{n}^{m}\left(\frac{r}{a}\right)^{n} Y_{n}^{m}(\theta, \phi)
$$

where $q_{n}^{m}$ are coefficients to be determined, and $N_{E}$ is the maximum spherical harmonic degree needed for the external fields. Since the magnetospheric sources are multiple Earth radii away from the satellite measurements, their fields can usually be well represented by a low-order spherical harmonic degree, such as 1 or 2 . The magnetic fields corresponding to the internal and external scalar potentials are

$$
\begin{aligned}
& \mathbf{M}\left(r, \theta, \phi ; g_{n}^{m}\right)=-\nabla V_{\text {int }}\left(r, \theta, \phi ; g_{n}^{m}\right)=\sum_{n m}\left(\frac{a}{r}\right)^{n+2} g_{n}^{m}\left[\begin{array}{c}
(n+1) Y_{n}^{m}(\theta, \phi) \\
-\partial_{\theta} Y_{n}^{m}(\theta, \phi) \\
-\frac{m}{\sin \theta} \partial_{\phi} Y_{n}^{m}(\theta, \phi)
\end{array}\right] \\
& \mathbf{K}\left(r, \theta, \phi ; q_{n}^{m}\right)=-\nabla V_{e x t}\left(r, \theta, \phi ; q_{n}^{m}\right)=-\sum_{n m}\left(\frac{r}{a}\right)^{n-1} q_{n}^{m}\left[\begin{array}{c}
n Y_{n}^{m}(\theta, \phi) \\
\partial_{\theta} Y_{n}^{m}(\theta, \phi) \\
\frac{m}{\sin \theta} \partial_{\phi} Y_{n}^{m}(\theta, \phi)
\end{array}\right]
\end{aligned}
$$

where the vector components are defined in geocentric spherical coordinates, ordered with respect to $r, \theta, \phi$. The magnetospheric ring current field is a primary contributor to $\mathbf{K}$, and since it is more efficiently parameterized in the solar magnetic (SM) coordinate system (Lühr et al. 2016), it is advantageous to expand the scalar potential $V_{\text {ext }}$ using SM coordinates, and then rotate the resulting $\mathbf{K}$ to geographic coordinates for fitting the satellite data. This is straightforward since the SM coordinate system is an Earth-centered Earth-fixed Euclidean system, which just involves a rotation from standard geocentric NEC coordinates. Similarly, the Sq field could be efficiently decomposed in QD coordinates, as it is aligned with the geomagnetic main field geometry at low and mid-latitudes, but great care must be taken when working with vector quantities in QD coordinates since they are a nonorthogonal system (Richmond 1995; Laundal and Richmond 2016) and that discussion goes beyond the scope of this chapter. Therefore, we will define the combined Sq and external field model to be fitted to the $F^{(1)}$ satellite residuals as

$$
\mathbf{T}\left(r, \theta, \phi ; g_{n}^{m}, q_{n}^{m}\right)=\mathbf{M}\left(r, \theta, \phi ; g_{n}^{m}\right)+\mathbf{K}\left(r, \theta^{S M}, \phi^{S M} ; q_{n}^{m}\right)
$$


where $\theta^{S M}$ and $\phi^{S M}$ are the colatitude and longitude of the point $(r, \theta, \phi)$ in SM coordinates. Equations for converting from geocentric spherical to SM coordinates may be found in Laundal and Richmond (2016). The Sq and external field model T can be fitted to the scalar residuals $F^{(1)}$ by minimizing the cost function

$$
\chi^{2}=\sum_{i}\left[F_{i}^{(1)}-\hat{\mathbf{b}}_{i} \cdot \mathbf{T}\left(\mathbf{r}_{i} ; \mathbf{x}\right)\right]^{2}+\lambda_{S q}^{2}\left\|L_{S q} \mathbf{x}\right\|^{2}
$$

where $\hat{\mathbf{b}}_{i}$ is a unit vector in the main field direction at the measurement point $\mathbf{r}_{i}, \mathbf{x}$ is a vector containing the model coefficients $g_{n}^{m}$ and $q_{n}^{m}, \lambda_{S q}$ is a regularization parameter, $L_{S q}$ is a regularization matrix, and $i$ is summed over all scalar residuals to be used in the Sq fitting for a particular orbit. The model fit is typically restricted to use data below about $60^{\circ}$ QD latitude to exclude effects from the high-latitude currents. Additionally one should exclude data in the EEJ region (between $\pm 12^{\circ}$ QD latitude), since the goal is to fit the large-scale Sq and external fields and preserve as much EEJ signal as possible. The model $\mathbf{T}$ is projected onto the main field direction in order to compare with the computed scalar field residuals $F_{i}^{(1)}$. Any standard main field model, such as IGRF (Thébault et al. 2015) or CHAOS (Finlay et al. 2016) could be used to compute $\hat{\mathbf{b}}_{i}$. When inverting scalar data over a single satellite orbit, it can be challenging to separate the internal $\mathrm{Sq}$ signal from the external magnetospheric signal, and so it is often useful to include the regularization term in the least-squares minimization. A typical choice for the regularization matrix is $L_{S q}=I$ to prevent the solution norm $\|\mathbf{x}\|$ from growing too large, resulting in nonphysical fields. However other choices could also work well if additional constraints are imposed on the internal and external fields (for example, minimizing latitudinal gradients of current flow). The regularization parameter $\lambda_{S q}$ represents a tradeoff between minimizing the model residuals and the solution norm. Choosing the right value of $\lambda_{S q}$ in an automated fashion can be a challenging problem, as the low and mid-latitude ionospheric and magnetospheric fields can change drastically during different local times, seasons, and geomagnetic activity levels. Selecting $\lambda_{S q}$ using L-curve analysis (Hansen and O'Leary 1993) often produces good results, although sometimes it is necessary to visually look at the data and fitted model to ensure a physically realistic fit is achieved. Once the model coefficients $g_{n}^{m}, q_{n}^{m}$ are determined for a particular satellite track, the Sq and external field contribution are removed from the residuals to isolate only the EEJ contribution to the ionospheric field. This is done by defining a new scalar residual

$$
F_{i}^{(2)}=F_{i}^{(1)}-\hat{\mathbf{b}}_{i} \cdot \mathbf{T}\left(\mathbf{r}_{i} ; g_{n}^{m}, q_{n}^{m}\right)
$$

The residuals $F^{(2)}$ will be used to invert for the EEJ current flow, discussed in the next section. Figure 11.2 (top panel) shows a single latitude profile recorded by Swarm B on 22 June 2015, when the satellite was in a 12:57 local time. The $F^{(1)}$ residual, computed by removing the core, crustal and magnetospheric field models from the scalar measurements is shown in purple as a function of QD latitude. At the magnetic equator, we see the characteristic sharp trough of the equatorial electrojet 

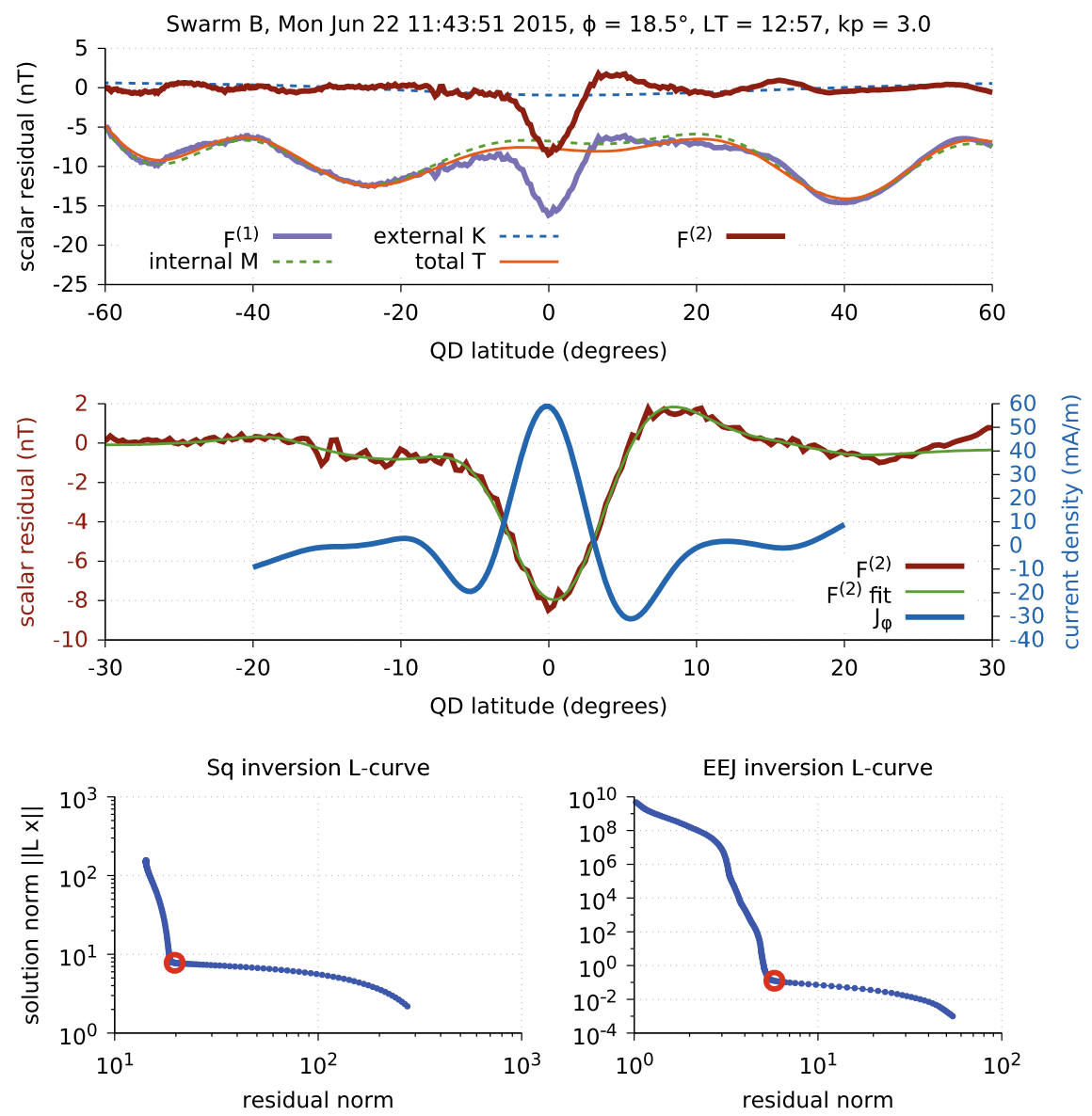

Fig. 11.2 Top: scalar residual $F^{(1)}$ (purple) with fitted model components $\mathbf{M}$ (dashed green), $\mathbf{K}$ (dashed blue), and total model $\mathbf{T}=\mathbf{M}+\mathbf{K}$ (orange) projected onto main field direction. Final residual $F^{(2)}$ plotted in red. Middle: Zoomed in view of $F^{(2)}$ residual (red) with fitted EEJ line current model (green) and equivalent E-region zonal current flow (blue). Bottom: L-curves from the fitted Sq/external model (left) and EEJ line current model (right). Computed L-curve corners, used to estimate regularization parameters, circled in red. Data recorded by Swarm B on 22 June 2015

signature. This data was recorded shortly before a strong geomagnetic storm and so the variations seen above $20^{\circ}$ QD latitude likely contain magnetospheric fields as well as the $\mathrm{Sq}$ signature. The result from fitting the internal and external source models $\mathbf{M}$ and $\mathbf{K}$ are shown as dashed green and blue lines, respectively. The internal $\mathbf{M}$ model closely tracks the higher latitude variations due to its degree 12 expansion in spherical harmonics. The external model $\mathbf{K}$ is expanded only to degree 1 in this calculation and so it exhibits only a large-scale slow variation with latitude. The combined model $\mathbf{T}$ is shown in orange and the final residual $F^{(2)}$ after removing the 
Sq and external models are shown in red. We see that the $F^{(2)}$ residual has close to zero mean at non-equatorial latitudes except for short-scale variations. This is a result of removing the background $\mathrm{Sq}$ field and leaving only the EEJ peak at the equator. The bottom left panel of Fig. 11.2 shows the L-curve, which is a log-log plot of the residual norm versus the solution norm of the least-squares function. The corner of the L-curve, circled in red, often represents a good tradeoff between minimizing the residuals and minimizing the solution norm (Hansen and O'Leary 1993). The remaining panels of the figure will be discussed in the next section.

\subsection{Estimating EEJ Flow with Line Currents}

The peak equatorial electrojet current flow follows the magnetic equator, since the horizontal field geometry enhances the zonal conductivity. Observationally, this was confirmed from CHAMP measurements (Lühr et al. 2004). Due to the magnetic eastward (or westward) flow of the EEJ, the spatial geometry of the current flow can be well represented by line currents. However, defining straight line currents tangent to the Earth at the longitude of the satellite's equator crossing would not take into account the Earth's curvature, nor would it account for the magnetic equator geometry. Therefore, we use instead small straight line segments, which can be placed along lines of constant magnetic latitude. Also, the endpoints of each segment can be fixed to a given E-region altitude to account for the spherical Earth geometry. Therefore, we define a set of $N_{C}$ "segmented" line currents following lines of constant quasi-dipole latitude at an altitude of $110 \mathrm{~km}$, and covering the low-latitude EEJ current flow region. The segmented line currents, hereafter referred to as simply line currents, are spaced apart at equal intervals in QD latitude. These line currents will represent equivalent height-integrated EEJ current flow, since the EEJ current system also has vertical structure which cannot be resolved by satellite measurements far above the current region. Figure 11.3 depicts the line current geometry along the magnetic equator. Each of the $N_{C}$ line currents is divided into 360 segments, with each segment spanning $1^{\circ}$ of longitude. The unit current vector for longitude segment $k \in[1, \ldots, 360]$ of line current $j \in\left[1, \ldots, N_{C}\right]$ is then given by

$$
\begin{aligned}
& I_{j k}^{r} \approx 0 \\
& I_{j k}^{\theta}=-\cos \alpha_{j k} \\
& I_{j k}^{\phi}=\sin \alpha_{j k}
\end{aligned}
$$

where $\alpha_{j k}$ is the angle between geographic north and the linear current segment $k$ of current $j$. The unit current vector $\mathbf{I}_{j k}$ defined above represents simply the spherical coordinate components of the direction of current flow for that particular line segment. Next, the unit magnetic field perturbation due to this single line segment is given by the Biot-Savart law: 


$$
d \mathbf{B}_{i j k}=\frac{\mu_{0}}{4 \pi} \delta_{j k} \frac{\mathbf{I}_{j k} \times\left(\mathbf{r}_{i}-\mathbf{r}_{j k}\right)}{\left|\mathbf{r}_{i}-\mathbf{r}_{j k}\right|^{3}}
$$

where $\delta_{j k}$ is the distance in meters of segment $k$ of line current $j, \mathbf{r}_{i}$ is the position vector of satellite observation $i$, and $\mathbf{r}_{j k}$ is the position vector pointing to the midpoint of line segment $k$ of line current $j$. The total magnetic field contribution from line current $j$ is simply the sum over all longitudinal segments $k$ :

$$
\mathbf{B}_{i j}=\sum_{k} d \mathbf{B}_{i j k}
$$

The sum is taken only over a subset of all 360 longitudinal segments, because current flowing far away from the satellite observation has less influence on the measured magnetic field. Experience has shown that only including contributions from segments within $\pm 30^{\circ}$ longitude of the satellite's equator crossing provide adequate estimates of the current strength. Next, we project $\mathbf{B}_{i j}$ onto the internal field direction using a main field model, since only the scalar field satellite measurements are used to fit the EEJ currents. The result is

$$
F_{i j}=\mathbf{B}_{i j} \cdot \hat{\mathbf{b}}_{i}
$$

Finally, assume the same current flows along each segment of one line current. Then there will be $j$ unknown line current strengths $s_{j}$ to determine from the magnetic measurements. These can be computed by minimizing the objective function

$$
\chi^{2}=\|\mathbf{b}-F \mathbf{s}\|^{2}+\lambda_{E E J}^{2}\left\|L_{E E J} \mathbf{s}\right\|^{2}
$$

where $\mathbf{b}$ is a vector of scalar field residuals $F_{i}^{(2)}, \mathbf{s}$ is a vector of length $N_{C}$ containing the line current strengths $s_{j}, \lambda_{E E J}$ is a regularization parameter, $L_{E E J}$ is a regularization matrix, and $F$ is the least-squares matrix relating the line current strengths to the scalar field observations, whose elements are the $F_{i j}$ defined in Eq. 11.16. The regularization term is added to prevent nonphysical solutions during the leastsquares inversion. The regularization matrix $L_{E E J}$ is typically set to a second order finite difference operator, to ensure a smooth variation along the latitudinal current profile and prevent neighboring currents from exhibiting large oscillations. The regularization parameter $\lambda_{E E J}$ provides a tradeoff between minimizing the residual norm $\|\mathbf{b}-F \mathbf{s}\|$ and the solution norm $\left\|L_{E E J} \mathbf{S}\right\|$. Similarly to the Sq field inversion, L-curve methods for determining $\lambda_{E E J}$ work well in practice. Figure 11.2 (middle panel) shows a zoomed-in view of the $F^{(2)}$ residual (red) computed by removing the $\mathrm{Sq}$ field, discussed in Sect. 11.3. The magnetic field of the line current model fitted to this residual is shown in green, while the line current profile is shown in blue. The line current profile was transformed into the geographic frame to show $J_{\phi}$ eastward flow. The L-curve for the EEJ line current inversion is shown in the bottom right panel, with the computed corner circled in red, which determined the regularization parameter for this fit. 


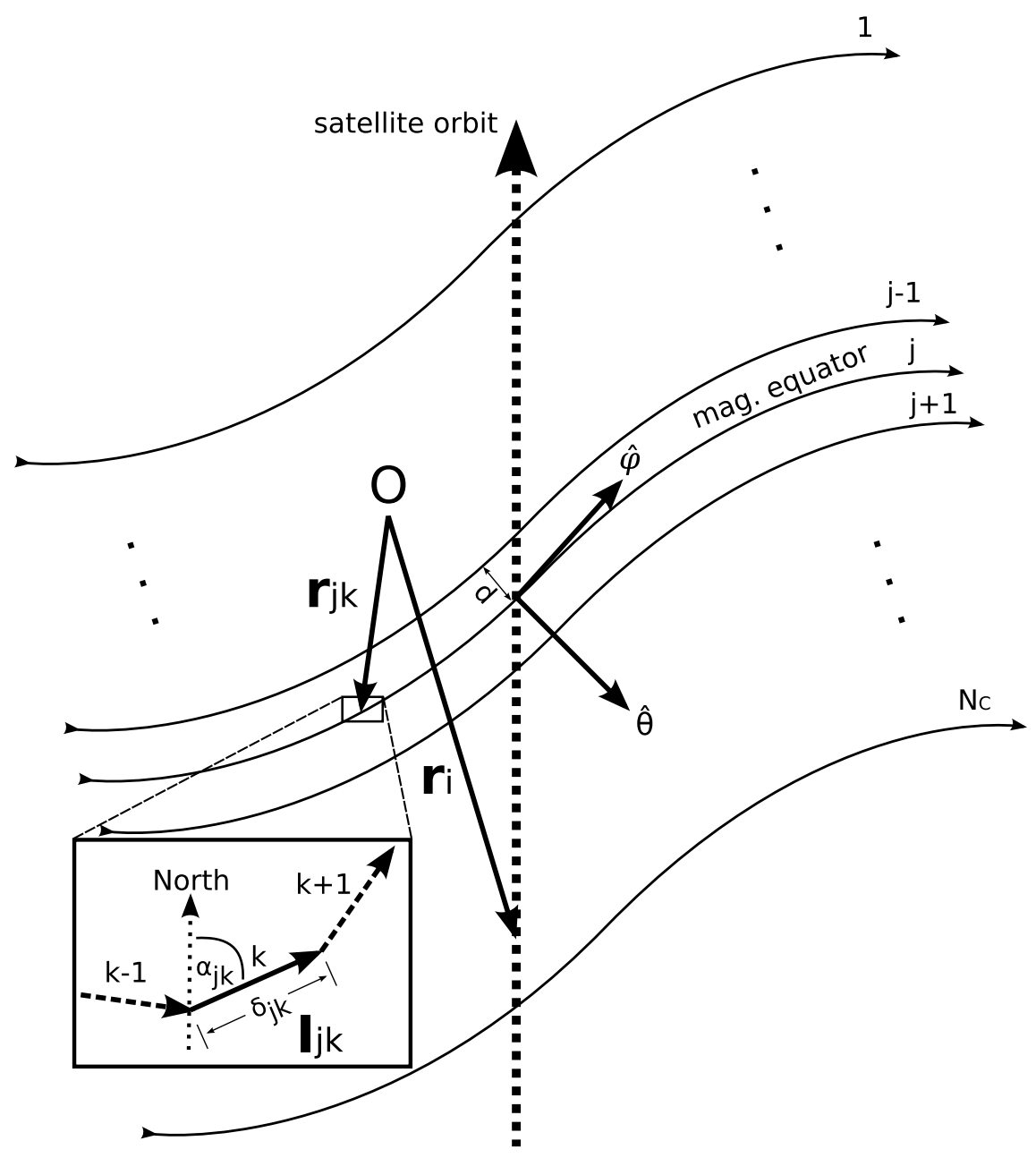

Fig. 11.3 Current model used for inversion. Currents $1 \ldots N_{C}$ shown following lines of constant quasi-dipole latitude, with the satellite crossing the magnetic equator (shown as current $j$ ). Origin $O$ represents the Earth's center with vector $\mathbf{r}_{j k}$ pointing to linear current segment $k$ of arc current $j$, and $\mathbf{r}_{i}$ pointing to satellite observation point $i$. Unit current vector $\mathbf{I}_{j k}$ shown in enlarged region with relevant parameters (see text). Basis vectors $\hat{\phi}$ and $\hat{\theta}$ are shown for use during the modeling step). Reproduced from Alken et al. (2013b)

The equatorial electrojet current strength is known to be modulated in longitude by atmospheric tides originating from latent heat release in deep convective tropical clouds. A number of different tidal components have been found to contribute to ionospheric longitudinal variability at low-latitudes, including the prominent eastward propagating diurnal tide with zonal wavenumber 3 (DE3) (Forbes et al. 2008; Lühr et al. 2008; Zhou et al. 2016). Figure 11.4 shows Swarm-derived height-integrated 


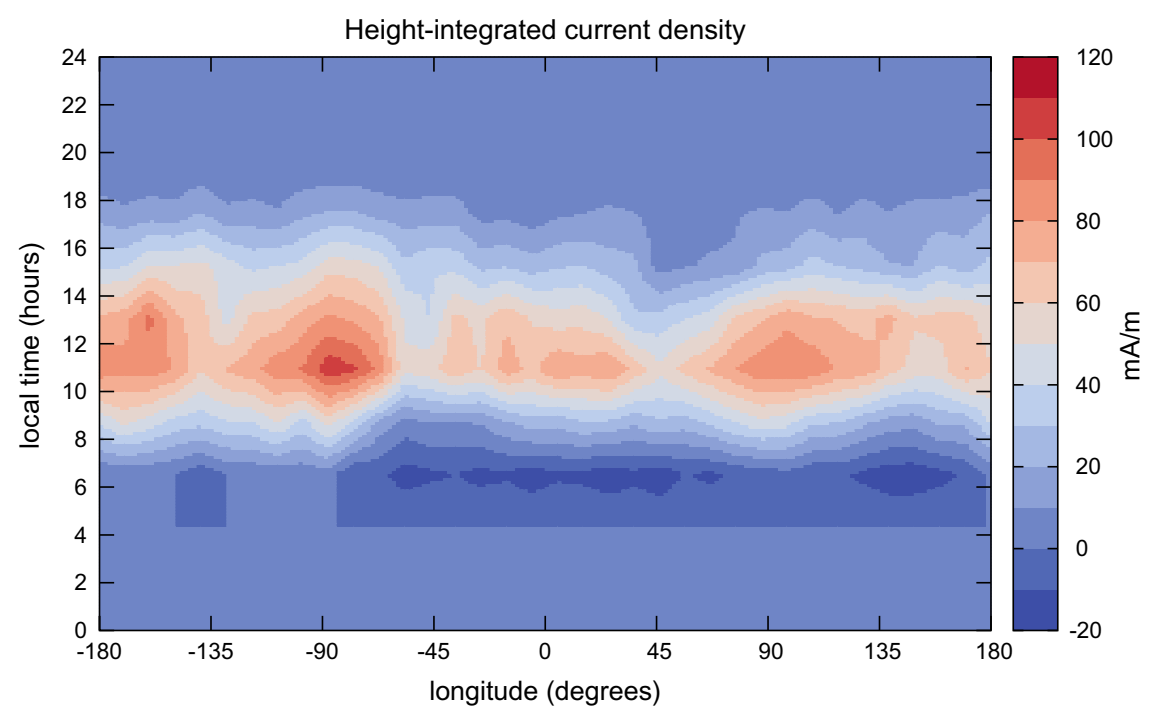

Fig. 11.4 Swarm derived height-integrated peak equatorial electrojet current for March equinox plotted as longitude versus local time

EEJ current estimates on the magnetic equator around the March equinox season and binned in longitude and local time. The evident wave- 4 structure in longitude is characteristic of the DE3 atmospheric tide. There are also other tidal components present in the EEJ dataset, which have been thoroughly analyzed by Lühr et al. (2008, 2012), Zhou et al. (2016), Yamazaki et al. (2017, 2018). It is also known that the tidal components exhibit a strong seasonal dependence. Notably for the EEJ, during the months of December and January the eastward propagating diurnal tide with zonal wavenumber 2 (DE2) becomes dominant over DE3. The figure also exhibits a westward flowing current around 0600 local time, indicated by the dark blue ribbon. This is known as counter electrojet (CEJ) and is often found in the early morning hours near the dawn terminator.

\subsection{Estimating Low-Latitude Electric Fields}

The current profile representing EEJ flow in the E-region discussed in the previous section contains a wealth of information about low-latitude ionospheric electrodynamics. Since the EEJ current system is affected by the neutral wind field and solar wind activity, the EEJ current derived from satellite observations can reveal a great deal about the underlying driving mechanisms, their seasonal and local time structure, and how they change during active and quiet times. Satellite-derived estimates of EEJ flow have been used to study atmospheric tides (Lühr and Manoj 2013; Zhou et al. 2016) and ionospheric response during geomagnetic storms (Astafyeva et al. 2016). 
We can go a step further, toward a more fundamental parameter than the currents themselves, which is the low-latitude electric field. The electric field is ultimately responsible for driving the currents, so knowledge of the electric field will enable a deeper understanding of the current system, as well as other low-latitude phenomena like the vertical plasma fountain (Anderson 1981; Stening 1992; Kelley 2009). In order to infer electric field information from satellite-derived currents, we need to apply some ionospheric modeling.

\subsubsection{Ionospheric Electrostatic Modeling}

We will ignore ionospheric dynamics which occur on time scales of one minute or less, which will allow us to assume a steady-state system, and thus consider only electrostatic fields. In this case, the equations governing the electric fields and currents are given by

$$
\begin{aligned}
\nabla \times \mathbf{E} & =0 \\
\mathbf{J} & =\sigma(\mathbf{E}+\mathbf{u} \times \mathbf{B})
\end{aligned}
$$

where $\mathbf{E}$ is the electrostatic field, $\mathbf{J}$ is the current density, $\sigma$ is the anisotropic conductivity tensor (Forbes 1981, Eq. 10), $\mathbf{u}$ is the neutral wind velocity field, and $\mathbf{B}$ is the ambient geomagnetic field. Equation (11.18) comes from Faraday's law assuming a steady-state magnetic field and Eq. (11.19) is Ohm's law describing the current density driven by the neutral winds and electric field. The main idea is to solve Eqs. (11.18), (11.19) for the electric field $\mathbf{E}$ in a low-latitude region by

1. making simplifying assumptions about the longitudinal structure of the current and electric fields in order to reduce from a $3 \mathrm{D}$ problem to $2 \mathrm{D}$

2. using global climatological models to specify the conductivity $\sigma$, neutral wind field $\mathbf{u}$, and geomagnetic field $\mathbf{B}$

3. using the satellite-derived current profile obtained by the methods of Sect. 11.4 to specify $J_{\phi}$, the eastward component of $\mathbf{J}$

First, we will ignore longitudinal gradients of the electric field and current density $(\partial \mathbf{E} / \partial \phi=\partial \mathbf{J} / \partial \phi=0)$. This assumption is known to be incorrect on large scales, as there are many reports in the literature of 3 and 4-cell patterns at low-latitudes in many ionospheric parameters, such as vertical plasma drift velocities, EEJ currents, and plasma density (Immel et al. 2006; England et al. 2006; Lühr et al. 2007, 2008, 2012) Gradients in $\mathbf{E} \times \mathbf{B}$ drift velocities have been reported up to $3 \mathrm{~m} / \mathrm{s} / \mathrm{deg}$ (AraujoPradere et al. 2011). To account for the full and complex longitude structure of the ionosphere, we would need to solve the electrostatic equations in three dimensions. However, by ignoring longitudinal gradients on local scales, the problem drastically simplifies, and previous studies calculating electric fields with this assumption have demonstrated remarkable agreement with independent radar measurements at Jicamarca (Alken and Maus 2010a; Alken et al. 2013a, 2015). This assumption, coupled 
with the divergence-free current condition, $\nabla \cdot \mathbf{J}=0$, allows the $J_{r}$ and $J_{\theta}$ current components to be derived from a single current stream function $\psi$ (Untiedt 1967; Sugiura and Poros 1969).

$$
\begin{aligned}
J_{r} & =\left(\frac{R}{r}\right)^{2} \frac{1}{\sin \theta} \frac{\partial \psi}{\partial \theta} \\
J_{\theta} & =-\left(\frac{R}{r}\right) \frac{R}{\sin \theta} \frac{\partial \psi}{\partial r}
\end{aligned}
$$

Equation (11.18) then becomes

$$
\begin{aligned}
& \partial_{r}\left(r E_{\theta}\right)-\partial_{\theta}\left(E_{r}\right)=0 \\
& \left.\begin{array}{rl}
\partial_{\theta}\left(\sin \theta E_{\phi}\right) & =0 \\
\partial_{r}\left(r E_{\phi}\right) & =0
\end{array}\right\} \Rightarrow E_{\phi}=\frac{R E_{\phi_{0}}}{r \sin \theta}
\end{aligned}
$$

where $R$ is a constant of integration and can be taken as a reference radius, and $E_{\phi_{0}}$ is the eastward electric field at the equator at the radius $R$. Equation (11.23) shows that for a given value of the equatorial eastward electric field $E_{\phi_{0}}=E_{\phi}(r=R, \theta=\pi / 2)$, $E_{\phi}(r, \theta)$ is determined everywhere in the $(r, \theta)$ plane. The unknowns to be determined are therefore $E_{r}, E_{\theta}$, and $\psi$.

Next, we use a priori climatological models to specify the conductivity $\sigma$, neutral wind field $\mathbf{u}$, and geomagnetic field $\mathbf{B}$. The conductivity requires knowledge of the global densities and temperatures of the electrons, ions and neutrals. For these we use the IRI-2012 (Bilitza et al. 2011) and NRLMSISE-00 (Picone et al. 2002) models. The equations for the direct, Pedersen and Hall conductivities are given in (Kelley 1989, Appendix B) and reproduced below:

$$
\begin{aligned}
\sigma_{0} & =e^{2}\left(\frac{n_{e}}{m_{e} v_{e}}+\sum_{i} \frac{n_{i}}{m_{i} v_{i}}\right) \\
\sigma_{p} & =e^{2}\left(\frac{n_{e} v_{e}}{m_{e}\left(v_{e}^{2}+\Omega_{e}^{2}\right)}+\sum_{i} \frac{n_{i} v_{i}}{m_{i}\left(v_{i}^{2}+\Omega_{i}^{2}\right)}\right) \\
\sigma_{h} & =e^{2}\left(\frac{n_{e} \Omega_{e}}{m_{e}\left(v_{e}^{2}+\Omega_{e}^{2}\right)}-\sum_{i} \frac{n_{i} \Omega_{i}}{m_{i}\left(v_{i}^{2}+\Omega_{i}^{2}\right)}\right)
\end{aligned}
$$

Here, the $i$ sums overall ion species in the ionosphere. $e$ is the electron charge, $n_{e}$ is the electron density, $n_{i}$ is the ion density of species $i, m_{e}$ and $m_{i}$ are the electron and ion masses, $v_{e}$ and $v_{i}$ are the electron and ion collision frequencies, and $\Omega_{e}$ and $\Omega_{i}$ are the electron and ion gyro-frequencies around the magnetic field lines. Expressions for the collision frequencies $v_{e}$ and $v_{i}$ are given in (Kelley 1989, Appendix B). The ionospheric densities and temperatures are taken from IRI-2012. The neutral density 
needed to compute the collision frequencies is taken from the NRLMSISE-00 model. Previous efforts to model the equatorial electrojet have found it necessary to increase the electron collision frequency $v_{e}$ by an empirical factor of 4 during typical daytime eastward electric field conditions, to account for unmodeled nonlinear instabilities in the electrojet stream (Gagnepain et al. 1977; Ronchi et al. 1990, 1991; Fang et al. 2008; Alken and Maus 2010a,b). We adopt this same convention when modeling satellite-derived EEJ profiles. The neutral wind field $\mathbf{u}$ is supplied by the Horizontal Wind Model (HWM14) (Drob et al. 2015). HWM14 does not provide vertical wind velocities, and so they are ignored during this modeling. Any standard geomagnetic field model, such as IGRF (Thébault et al. 2015) can be used to specify B.

Eliminating $E_{r}$ and $E_{\theta}$ from Eqs. (11.19)-(11.22) yields a second order partial differential equation (PDE) for the current stream function $\psi$ :

$$
f_{1} \frac{\partial^{2} \psi}{\partial r^{2}}+2 f_{2} \frac{\partial^{2} \psi}{\partial r \partial \theta}+f_{3} \frac{\partial^{2} \psi}{\partial \theta^{2}}+f_{4} \frac{\partial \psi}{\partial r}+f_{5} \frac{\partial \psi}{\partial \theta}=g
$$

where the coefficients $f_{1}, \ldots, f_{5}$ and right hand side $g$ are functions of $r, \theta$ given by

$$
\begin{aligned}
f_{1}= & r^{2} \sigma_{r r} \\
f_{2}= & \frac{1}{2} r\left(\sigma_{r \theta}+\sigma_{\theta r}\right) \\
f_{3}= & \sigma_{\theta \theta} \\
f_{4}= & r \alpha\left[r \frac{\partial}{\partial r}\left(\frac{\sigma_{r r}}{\alpha}\right)+\sin \theta \frac{\partial}{\partial \theta}\left(\frac{1}{\sin \theta} \frac{\sigma_{r \theta}}{\alpha}\right)\right] \\
f_{5}= & \alpha\left[r^{2} \frac{\partial}{\partial r}\left(\frac{1}{r} \frac{\sigma_{\theta r}}{\alpha}\right)+\sin \theta \frac{\partial}{\partial \theta}\left(\frac{1}{\sin \theta} \frac{\sigma_{\theta \theta}}{\alpha}\right)\right] \\
g= & \frac{r}{R} \alpha\left[r \frac{\partial}{\partial r}\left(\frac{\gamma}{\alpha}\right)+\sin \theta \frac{\partial}{\partial \theta}\left(\frac{1}{\sin \theta} \frac{\beta}{\alpha}\right)\right] E_{\phi_{0}}+ \\
& \left(\frac{r}{R}\right)^{2} \alpha \sin \theta\left\{\frac{\partial}{\partial r}\left[\frac{r}{\alpha}\left(\sigma_{\theta r} W_{r}-\sigma_{r r} W_{\theta}\right)\right]+\frac{\partial}{\partial \theta}\left[\frac{1}{\alpha}\left(\sigma_{\theta \theta} W_{r}-\sigma_{r \theta} W_{\theta}\right)\right]\right\}
\end{aligned}
$$

with

$$
\begin{aligned}
& \alpha=\sigma_{r r} \sigma_{\theta \theta}-\sigma_{r \theta} \sigma_{\theta r} \\
& \beta=\sigma_{\theta \theta} \sigma_{r \phi}-\sigma_{r \theta} \sigma_{\theta \phi} \\
& \gamma=\sigma_{r \phi} \sigma_{\theta r}-\sigma_{r r} \sigma_{\theta \phi}
\end{aligned}
$$

and $\mathbf{W}=\sigma(\mathbf{u} \times \mathbf{B})$. The conductivity tensor $\sigma$ is represented in a basis of spherical coordinates. The components of the conductivity tensor may be related to the direct, Pedersen, and Hall conductivities using (Richmond 1995, Eq. 2.1), or alternatively 
in coordinate-free matrix notation:

$$
\sigma=\sigma_{p} I+\left(\sigma_{0}-\sigma_{p}\right) \mathbf{b b}^{T}+\sigma_{h}[\mathbf{b}]_{\times}
$$

where $\mathbf{b}$ is a unit vector in the geomagnetic field direction $\mathbf{B}$ and $[\mathbf{b}]_{\times}$is the skewsymmetric matrix defined by the cross product with b. In spherical coordinates, $\mathbf{b}=\left(b_{r}, b_{\theta}, b_{\phi}\right)^{T}$ and

$$
[\mathbf{b}]_{\times}=\left(\begin{array}{ccc}
0 & -b_{\phi} & b_{\theta} \\
b_{\phi} & 0 & -b_{r} \\
-b_{\theta} & b_{r} & 0
\end{array}\right)
$$

We solve Eq. (11.27) in spherical geocentric coordinates, however the coordinates are rotated so that the azimuthal direction $\hat{\phi}$ is tangent to the magnetic equator at the location of the satellite crossing (see Fig. 11.3). This is a first-order correction in order to allow the modeling of the currents flowing along lines of constant quasidipole latitude, as we calculated during the satellite data inversion step. To perform a strict comparison between our modeled current and satellite-derived current would require solving the electrostatic equations in quasi-dipole coordinates, but this firstorder correction enables us to use the simplicity of spherical coordinates and captures most of the difference between magnetic and geographic east. The PDE is solved on a $2 \mathrm{D}$ grid in the $(r, \theta)$ plane, holding the longitude $\phi$ fixed where the satellite crosses the magnetic equator. The grid should be large enough to encompass the main current flow at low-latitudes, including the meridional current system, but not so large to include effects of current flow from mid and high-latitudes. Typically, a grid ranging from 65 to $500 \mathrm{~km}$ altitude, and $-25^{\circ}$ to $25^{\circ}$ latitude is sufficient for capturing the main EEJ current of interest. The boundary conditions on the PDE are that the current should vanish at the lower and upper boundaries $\left(\psi=0\right.$ at $r=r_{\min }$ and $\left.r=r_{\text {max }}\right)$, and there is no radial current flow at the northern and southern boundaries $\left(\partial_{\theta} \psi=0\right.$ at $\theta=\theta_{\min }$ and $\left.\theta=\theta_{\max }\right)$. The PDE can be solved with standard finite difference methods.

\subsubsection{Estimating the Electric Field}

The solution for the current stream function $\psi$ depends on the conductivities, neutral wind field, and geomagnetic field components in the solution region, but it also requires a value for the eastward electric field component on the magnetic equator, $E_{\phi_{0}}$. The former fields are specified by climatological models, but the eastward electric field is what we wish to obtain as a final output of this procedure. There is one additional piece of information we have not yet used, which is the satellitederived zonal current profile. The solution for the electric field now becomes an optimization problem: which value of $E_{\phi_{0}}$ produces a modeled current which best agrees with the satellite-derived current? One approach would be to solve Eq. (11.27) 
multiple times with different values of $E_{\phi_{0}}$ until we obtain the best agreement between the modeled and observed current. However, in this case we are fortunate that the current density $\mathbf{J}$ in Eq. (11.19) is a linear function of the electric field $\mathbf{E}$ when $\mathbf{u}=0$. Therefore we only need to solve the PDE twice, once with the full wind field $\mathbf{u}$, and a second time with $\mathbf{u}=0$. Then the unknown component $E_{\phi_{0}}$ can be determined from a least-squares inversion of

$$
\begin{aligned}
J_{\phi}^{S A T}(\theta) & =s J_{P D E}\left(\theta ; E_{\phi_{0}}=1 \mathrm{mV} / \mathrm{m}, \mathbf{u}=0\right) \\
& +J_{P D E}\left(\theta ; E_{\phi_{0}}=0, \mathbf{u}\right) \\
& -J_{D C}
\end{aligned}
$$

where $s$ is a scaling factor (discussed below), $J_{\phi}^{S A T}(\theta)$ is the latitude current profile derived from the satellite observations, $J_{P D E}(\theta)$ is the height-integrated zonal current profile calculated from the PDE solution:

$$
J_{P D E}(\theta)=\sum_{i} J_{\phi}\left(r_{i}, \theta\right) \delta r
$$

where $\delta r$ is the radial grid spacing and $J_{\phi}(r, \theta)$ is the PDE-derived zonal current on the $(r, \theta)$ grid, and finally $J_{D C}$ is a constant offset to allow for a difference in zerolevels between the modeled and observed current. As can be seen in Eq. (11.34), the PDE is solved twice, once with a "unit" value of $E_{\phi_{0}}=1 \mathrm{mV} / \mathrm{m}$ with the wind field turned off, and a second time with $E_{\phi_{0}}=0$ with the wind field turned on. The parameters $s$ and $J_{D C}$ are determined by least-squares inversion of Eq. (11.34) with the additional constraint that the left and right hand sides of that equation must agree on the magnetic equator $(\theta=\pi / 2)$. This constraint has been found to yield more accurate electric fields (Alken and Maus 2010a; Alken et al. 2013b, 2015), since the EEF is primarily responsible for current flow near the magnetic equator, while the neutral winds affect current flow at higher latitudes (Fambitakoye and Mayaud 1976).

Figure 11.5 shows the height-integrated current density profile derived from Swarm B near 12 UT on 1 January 2017 (red) along with the modeled current density profile (green). For this orbit, the satellite was in local time of 13:06. The two current profiles agree well at the magnetic equator, which was a condition imposed during the least-squares inversion. We can see that the main peak is modeled well, while the side-lobes differ considerably. This is fairly typical in our modeling approach, since the side-lobes are mainly determined by the neutral winds at higher altitudes (Fambitakoye and Mayaud 1976), and the climatological wind model (HWM14) cannot capture the highly variable winds. However, as discussed by Fambitakoye and Mayaud (1976), Reddy and Devasia (1981), the height and width of the main peak is primarily determined by the EEF, and so an accurate model of this peak will produce reliable estimates of the eastward equatorial electric field component.

Figure 11.6 shows the eastward equatorial electric field data derived from four years of Swarm data (2014-2017), selected for March equinox season and binned 


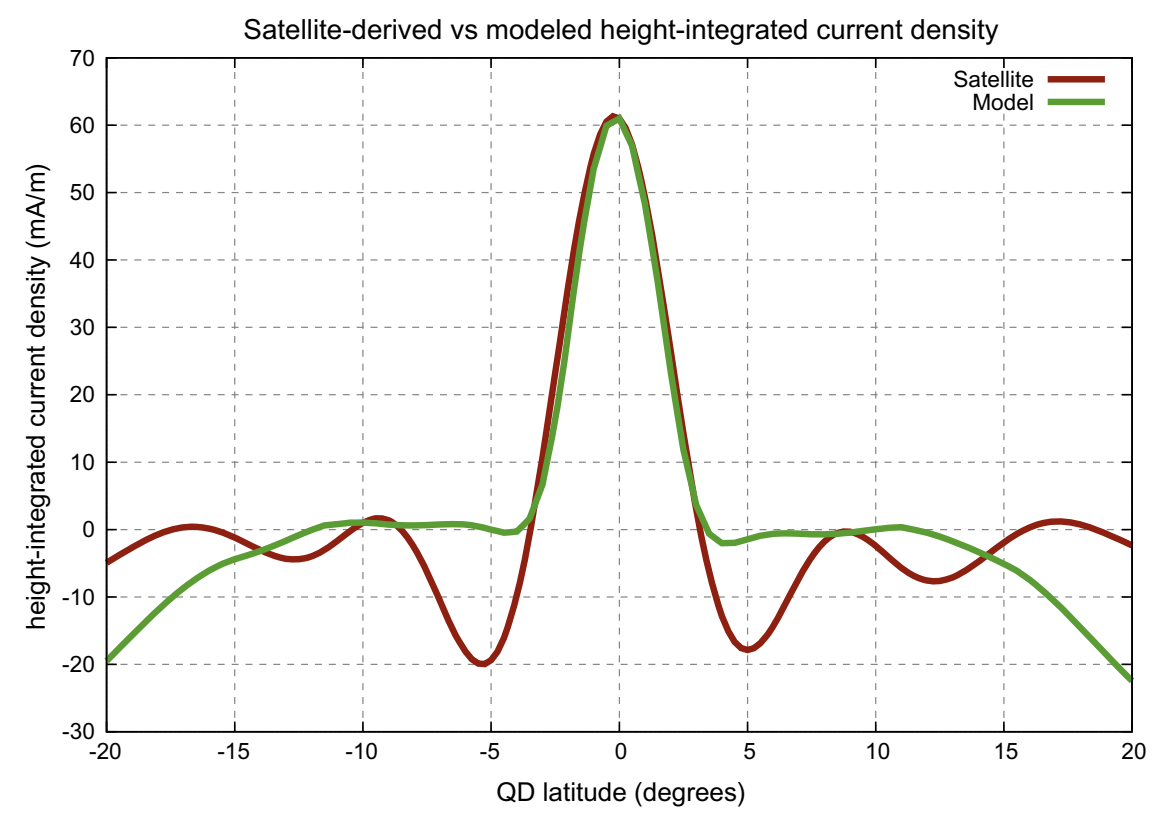

Fig. 11.5 Height-integrated EEJ current density derived from Swarm B measurements near 12 UT on 1 January 2017 in red. Corresponding modeled current density is shown in green

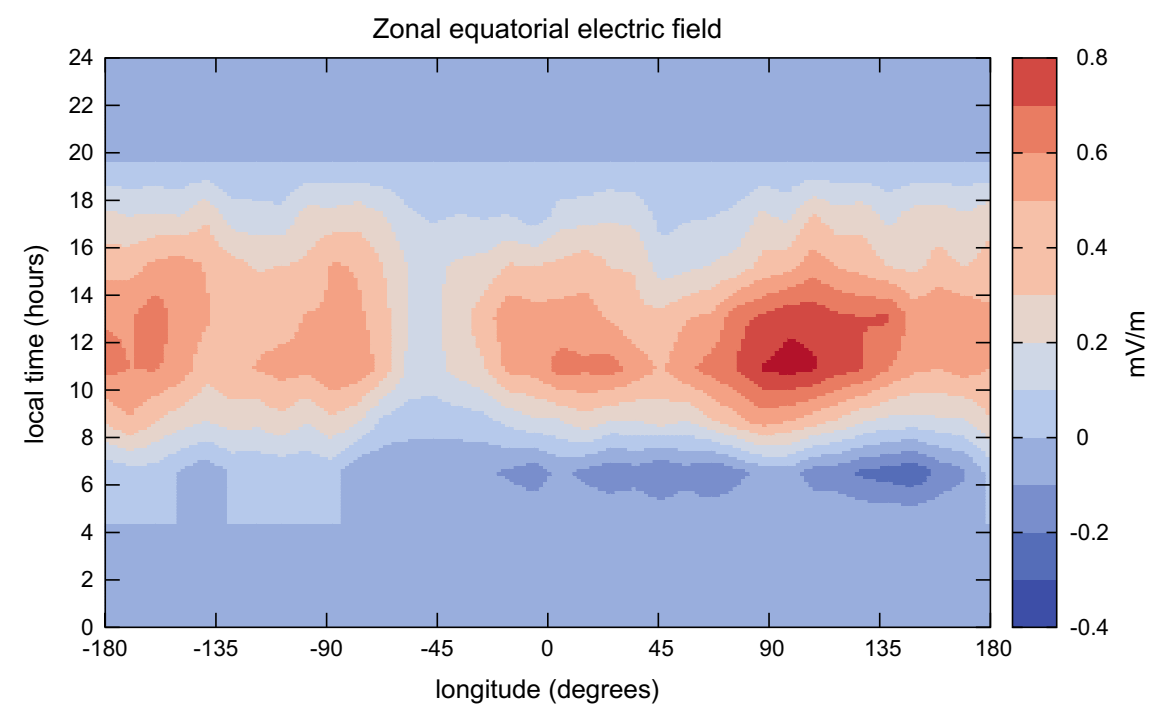

Fig. 11.6 Swarm derived zonal equatorial electric field for March equinox, plotted as longitude versus local time 
as a function of longitude and local time. Similar to Fig. 11.4 we see the wave-4 longitude structure due to the DE3 tide, as well as the westward electric field around 0600 local time indicating a counter electrojet.

\subsection{Conclusion}

We have presented a methodology of inverting scalar magnetic measurements from LEO near-polar orbiting satellites for EEJ equivalent current flow at low-latitudes. This method is based on modeling the magnetically eastward current flow with straight line segments placed along lines of constant quasi-dipole latitude. The line currents are spaced equidistantly in QD latitude, and they have simple expressions for their magnetic field perturbations which can be fit to the satellite measurements using regularized least-squares methods. The result of this least-squares inversion is a latitude profile of zonal current flow at low-latitudes which corresponds to heightintegrated equivalent EEJ current in the E-region. These latitude profiles can further be used to recover the eastward component of the equatorial electric field by using a physics-based modeling procedure which relies on input from global climatological models to specify the state of the ionosphere and neutral atmosphere in the vicinity of the satellite measurements. Both the equivalent current profiles and the EEF estimates can be used to study seasonal and local time variations of the low-latitude ionosphere, as well as tidal characteristics of the low-latitude neutral atmosphere.

Acknowledgements The European Space Agency (ESA) is gratefully acknowledged for providing Swarm data and for financially supporting the Swarm Level-2 equatorial electric field (EEF) product. We thank the International Space Science Institute (ISSI) in Bern, Switzerland for supporting the working group "Ionospheric multi-satellite analysis tools" from which this chapter resulted. The editors thank an anonymous reviewer for his assistance in evaluating this chapter.

\section{References}

Aakjær, C.D., N. Olsen, and C.C. Finlay. 2016. Determining polar ionospheric electrojet currents from Swarm satellite constellation magnetic data. Earth, Planets and Space 68 (1): 140. https:// doi.org/10.1186/s40623-016-0509-y.

Alken, P., and S. Maus. 2010a. Electric fields in the equatorial ionosphere derived from CHAMP satellite magnetic field measurements. Journal of Atmospheric and Solar-Terrestrial Physics 72: 319-326. https://doi.org/10.1016/j.jastp.2009.02.006.

Alken, P., and S. Maus. 2010b. Relationship between the ionospheric eastward electric field and the equatorial electrojet. Geophysical Research Letters 37: L04104. https://doi.org/10.1029/ 2009GL041989.

Alken, P., A. Chulliat, and S. Maus. 2013a. Longitudinal and seasonal structure of the ionospheric equatorial electric field. Journal of Geophysical Research 118. https://doi.org/10.1029/ 2012JA018314. 
Alken, P., S. Maus, P. Vigneron, O. Sirol, and G. Hulot. 2013b. Swarm SCARF equatorial electric field inversion chain. Earth, Planets and Space 65 (11): 1309-1317. https://doi.org/10.5047/eps. 2013.09.008.

Alken, P., S. Maus, H. Lühr, R.J. Redmon, F. Rich, B. Bowman, and S.M. O’Malley. 2014. Geomagnetic main field modeling with DMSP. Journal of Geophysical Research 119. https://doi.org/ 10.1002/2013JA019754.

Alken, P., S. Maus, A. Chulliat, P. Vigneron, O. Sirol, and G. Hulot. 2015. Swarm equatorial electric field chain: first results. Geophysical Research Letters 42 (3): 673-680. https://doi.org/10.1002/ 2014GL062658,2014GL062658.

Anderson, D.N. 1981. Modeling the ambient, low latitude F-region ionosphere-A review. Journal of Atmospheric and Solar-Terrestrial Physics 43 (8): 753-762.

Araujo-Pradere, E.A., D.N. Anderson, and M. Fedrizzi. 2011. Communications/navigation outage forecasting system observational support for the equatorial E x B drift velocities associated with the four-cell tidal structures. Radio Science 46:RS0D09. https://doi.org/10.1029/2010RS004557.

Astafyeva, E., I. Zakharenkova, and P. Alken. 2016. Prompt penetration electric fields and the extreme topside ionospheric response to the 22-23 June 2015 geomagnetic storm as seen by the Swarm constellation. Earth, Planets and Space 68(152). https://doi.org/10.1186/s40623-0160526-X.

Bilitza, D., L.A. McKinnell, B. Reinisch, and T. Fuller-Rowell. 2011. The International Reference Ionosphere (IRI) today and in the future. Journal of Geodesy 85: 909-920. https://doi.org/10. 1007/s00190-010-0427-x.

Drob, D.P., J.T. Emmert, J.W. Meriwether, J.J. Makela, E. Doornbos, M. Conde, G. Hernandez, J. Noto, K.A. Zawdie, S.E. McDonald, J.D. Huba, and J.H. Klenzing. 2015. An update to the Horizontal Wind Model (HWM): The quiet time thermosphere. Earth and Space Science 2 (7): 301-319. https://doi.org/10.1002/2014EA000089,2014EA000089.

England, S.L., S. Maus, T.J. Immel, S.B. Mende. 2006. Longitudinal variation of the E-region electric fields caused by atmospheric tides. Geophysical Research Letters 33.

Fambitakoye, O., and P.N. Mayaud. 1976. The equatorial electrojet and regular daily variation $S_{R}$ :-I. A determination of the equatorial electrojet parameters. Journal of Atmospheric and Terrestrial Physics 38: 1-17.

Fang, T.W., A.D. Richmond, J.Y. Liu, A. Maute, C.H. Lin, C.H. Chen, and B. Harper. 2008. Model simulation of the equatorial electrojet in the Peruvian and Philippine sectors. Journal of Atmospheric and Solar-Terrestrial Physics 70: 2203-2211.

Finlay, C.C., N. Olsen, S. Kotsiaros, N. Gillet, and L. Tøffner-Clausen. 2016. Recent geomagnetic secular variation from Swarm and ground observatories as estimated in the CHAOS-6 geomagnetic field model. Earth, Planets and Space 68 (1): 112. https://doi.org/10.1186/s40623-0160486-1.

Fiori, R.A.D., and D.H. Boteler. 2018. Spherical cap Harmonic analysis techniques for mapping high-latitude ionospheric plasma flow-application to the Swarm mission. Submitted.

Forbes, J.M. 1981. The equatorial electrojet. Reviews of Geophysics and Space Physics 19 (3): 469-504.

Forbes, J.M., X. Zhang, S. Palo, J. Russell, C.J. Mertens, and M. Mlynczak. 2008. Tidal variability in the ionospheric dynamo region. Journal of Geophysical Research 113: A02310. https://doi. org/10.1029/2007JA012737.

Friis-Christensen, E., H. Lühr, and G. Hulot. 2006. Swarm: A constellation to study the Earth's magnetic field. Earth, Planets and Space 58: 351-358.

Gagnepain, J., M. Crochet, and A.D. Richmond. 1977. Comparison of equatorial electrojet models. Journal of Atmospheric and Terrestrial Physics 39: 1119-1124.

Hansen, P.C., and D.P. O'Leary. 1993. The use of the L-Curve in the regularization of discrete Ill-posed problems. SIAM Journal on Scientific Computing 14(6):1487-1503. https://doi.org/10. $1137 / 0914086$. 
Immel, T.J., E. Sagawa, S.L. England, S.B. Henderson, M.E. Hagan, S.B. Mende, H.U. Frey, C.M. Swenson, and L.J. Paxton. 2006. Control of equatorial ionospheric morphology by atmospheric tides. Geophysical Research Letters 33(L15108). https://doi.org/10.1029/2006GL026161.

Kelley, M.C. 1989. The Earth's ionosphere: Plasma physics and electrodynamics., International Geophysics Series San Diego: Academic Press Inc.

Kelley, M.C. 2009. The Earth's ionosphere: Plasma physics and electrodynamics., International Geophysics Series San Diego: Academic Press Inc.

Laundal, K.M., and A.D. Richmond. 2016. Magnetic coordinate systems. Space Science Reviews $1-33$.

Lühr, H., and C. Manoj. 2013. The complete spectrum of the equatorial electrojet related to solar tides: CHAMP observations. Annales de Geophysicae 31: 1315-1331.

Lühr, H., and S. Maus. 2010. Solar cycle dependence of quiet-time magnetospheric currents and a model of their near-Earth magnetic fields. Earth, Planets and Space 62: 843-848.

Lühr, H., S. Maus, and M. Rother. 2004. Noon-time equatorial electrojet: Its spatial features as determined by the CHAMP satellite. Journal of Geophysical Research 109: A01306. https://doi. org/10.1029/2002JA009656.

Lühr, H., K. Häusler, and C. Stolle. 2007. Longitudinal variation of F region electron density and thermospheric zonal wind caused by atmospheric tides. Geophysical Research Letters 34(16):L16102. https://doi.org/10.1029/2007GL030639,

Lühr, H., M. Rother, K. Häusler, P. Alken, and S. Maus. 2008. The influence of non-migrating tides on the longitudinal variation of the equatorial electrojet. Journal of Geophysical Research 113: A08313. https://doi.org/10.1029/2008JA013064.

Lühr, H., M. Rother, K. Häusler, B. Fejer, and P. Alken. 2012. Direct comparison of nonmigrating tidal signatures in the electrojet, vertical plasma drift and equatorial ionization anomaly. Journal of Atmospheric and Solar-Terrestrial Physics 75-76: 31-43. https://doi.org/10.1016/j.jastp.2011. 07.009 .

Lühr, H., C. Xiong, N. Olsen, G. Le. 2016. Near-Earth magnetic field effects of large-scale magnetospheric currents. Space Science Reviews 1-25. https://doi.org/10.1007/s11214-0160267-y.

Maus, S., and H. Lühr. 2005. Signature of the quiet-time magnetospheric magnetic field and its electromagnetic induction in the rotating Earth. Geophysical Journal International 162: 755763. https://doi.org/10.1111/j.1365-246X.2005.02691.x.

Maus, S., F. Yin, H. Lühr, C. Manoj, M. Rother, J. Rauberg, I. Michaelis, C. Stolle, and R.D. Müller. 2008. Resolution of direction of oceanic magnetic lineations by the sixth-generation lithospheric magnetic field model from CHAMP satellite magnetic measurements. Geochemistry, Geophysics, Geosystems 9(7):Q07021. https://doi.org/10.1029/2008GC001949.

Maus, S., C. Manoj, J. Rauberg, I. Michaelis, and H. Lühr. 2010. NOAA/NGDC candidate models for the 11th generation international geomagnetic reference field and the concurrent release of the 6th generation Pomme magnetic model. Earth, Planets and Space 62: 729-735.

Olsen, N. 1996. A new tool for determining ionospheric currents from magnetic satellite data. Geophysical Research Letters 23 (24): 3635-3638. https://doi.org/10.1029/96GL02896.

Picone, J.M., A.E. Hedin, D.P. Drob, and A.C. Aikin. 2002. NRLMSISE-00 empirical model of the atmosphere: statistical comparisons and scientific issues. Journal of Geophysical Research 107. https://doi.org/10.1029/2002JA009430.

Reddy, C.A., and C.V. Devasia. 1981. Height and latitude structure of electric fields and currents due to local eastwest winds in the equatorial electrojet. Journal of Geophysical Research: Space Physics 86 (A7): 5751-5767. https://doi.org/10.1029/JA086iA07p05751.

Richmond, A.D. 1995. Ionospheric electrodynamics using magnetic apex coordinates. Journal of Geomagnetism and Geoelectricity 47: 191-212.

Ritter, P., H. Lühr, A. Viljanen, O. Amm, A. Pulkkinen, and I. Sillanpää. 2004. Ionospheric currents estimated simultaneously from CHAMP satelliteand IMAGE ground-based magnetic field measurements: a statisticalstudy at auroral latitudes. Annales Geophysicae 22 (2): 417-430. 
Ronchi, C., R.N. Sudan, and P.L. Similon. 1990. Effect of short-scale turbulence on kilometer wavelength irregularities in the equatorial electrojet. Journal of Geophysical Research 95 (A1): 189-200.

Ronchi, C., R.N. Sudan, D.T. Farley. 1991. Numerical simulations of large-scale plasma turbulence in the daytime equatorial electrojet. Journal of Geophysical Research 96(A12):21,263-21,279.

Stening, R. 1992. Modelling the low latitude F region. Journal of Atmospheric and Terrestrial Physics 54(1112):1387-1412. https://doi.org/10.1016/0021-9169(92)90147-D, url http://www. sciencedirect.com/science/article/pii/002191699290147D.

Sugiura, M., and D.J. Poros. 1969. An improved model equatorial electrojet with a meridional current system. Journal of Geophysical Research 74: 4025-34.

Thébault, E., C.C. Finlay, C.D. Beggan, P. Alken, J. Aubert, O. Barrois, F. Bertrand, T. Bondar, A. Boness, L. Brocco, E. Canet, A. Chambodut, A. Chulliat, P. Coïsson, F. Civet, A. Du, A. Fournier, I. Fratter, N. Gillet, B. Hamilton, M. Hamoudi, G. Hulot, T. Jager, M. Korte, W. Kuang, X. Lalanne, B. Langlais, J.M. Léger, V. Lesur, F.J. Lowes, S. Macmillan, M. Mandea, C. Manoj, S. Maus, N. Olsen, V. Petrov, V. Ridley, M. Rother, T.J. Sabaka, D. Saturnino, R. Schachtschneider, O. Sirol, A. Tangborn, A. Thomson, L. Tøffner-Clausen, P. Vigneron, I. Wardinski, and T. Zvereva. 2015. International geomagnetic reference field: The 12th generation. Earth, Planets and Space 67(79). https://doi.org/10.1186/s40623-015-0228-9.

Untiedt, J. 1967. A model of the equatorial electrojet involving meridional currents. Journal of Geophysical Research 72(23).

Vanhamäki, H., L. Juusola, K. Kauristie, A. Workayehu, and S. Käki. 2018. Spherical elementary current systems applied to Swarm data. Submitted.

Vennerstrom, S., and T. Moretto. 2013. Monitoring auroral electrojets with satellite data. Space Weather 11 (9): 509-519. https://doi.org/10.1002/swe.20090.

Yamazaki, Y., C. Stolle, J. Matzka, T. Siddiqui, H. Lühr, and P. Alken. 2017. Longitudinal variation of the lunar tide in the equatorial electrojet. Journal of Geophysical Research 122(12). https:// doi.org/10.1002/2017JA024601.

Yamazaki, Y., C. Stolle, J. Matzka, and P. Alken. 2018. Quasi-6 day wave modulation of the equatorial electrojet. Journal of Geophysical Research 123. https://doi.org/10.1029/2018JA025365.

Zhou, Y., H. Lühr, P. Alken, and C. Xiong. 2016. New perspectives on equatorial electrojet tidal characteristics derived from the Swarm constellation. Journal of Geophysical Research: Space Physics 121. https://doi.org/10.1002/2016JA022713.

Open Access This chapter is licensed under the terms of the Creative Commons Attribution 4.0 International License (http://creativecommons.org/licenses/by/4.0/), which permits use, sharing, adaptation, distribution and reproduction in any medium or format, as long as you give appropriate credit to the original author(s) and the source, provide a link to the Creative Commons license and indicate if changes were made.

The images or other third party material in this chapter are included in the chapter's Creative Commons license, unless indicated otherwise in a credit line to the material. If material is not included in the chapter's Creative Commons license and your intended use is not permitted by statutory regulation or exceeds the permitted use, you will need to obtain permission directly from the copyright holder.

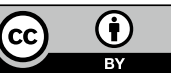

